TRANSACTIONS OF THE

AMERICAN MATHEMATICAL SOCIETY

Volume 357, Number 8, Pages 3119-3131

S 0002-9947(04)03765-1

Article electronically published on November 4, 2004

\title{
A COUNTABLE TEICHMÜLLER MODULAR GROUP
}

\author{
KATSUHIKO MATSUZAKI
}

\begin{abstract}
We construct an example of a Riemann surface of infinite topological type for which the Teichmüller modular group consists of only a countable number of elements. We also consider distinguished properties which the Teichmüller space of this Riemann surface possesses.
\end{abstract}

\section{$\S 1$. INTRODUCTION}

In this paper, we construct an example of a Riemann surface of infinite topological type for which the Teichmüller modular group has only a countable number of elements. The Teichmüller space of such a Riemann surface is infinite dimensional. For an analytically finite Riemann surface, its Teichmüller space is finite dimensional and the Teichmüller modular group is finitely generated, in particular countable. Hence our example proves the existence of an infinite-dimensional Teichmüller space that has a nature of finite dimension regarding the cardinality of the modular group. This answers a problem raised by Epstein [3].

Actually, the original problem asks whether the reduced Teichmüller modular group, which is the quotient group of the Teichmüller modular group by free homotopy equivalence, can be countable or not. By making our Riemann surface $R$ have no ideal boundary at infinity, we give a stronger example where even the full Teichmüller modular group $\operatorname{Mod}(R)$ is countable. Then we consider certain properties which the Teichmüller space $T(R)$ of this $R$ has. For instance, we prove discontinuity of the action of $\operatorname{Mod}(R)$ on $T(R)$ and triviality of the action of $\operatorname{Mod}(R)$ at the origin of the asymptotic Teichmüller space $A T(R)$. The latter space has been studied by Earle, Gardiner and Lakic [4].

Throughout this paper, we assume that a Riemann surface $R$ is hyperbolic, that is, it is represented as a quotient space $\mathbb{H}^{2} / \Gamma$ of the hyperbolic plane $\mathbb{H}^{2}$ by a torsion free Fuchsian group $\Gamma$. The Teichmüller space $T(R)$ of $R$ is the set of all equivalence classes of the pair $(f, \sigma)$, where $f: R \rightarrow R_{\sigma}$ is a quasiconformal homeomorphism of $R$ onto another Riemann surface $R_{\sigma}$ of a complex structure $\sigma$. Two pairs $\left(f_{1}, \sigma_{1}\right)$ and $\left(f_{2}, \sigma_{2}\right)$ are defined to be equivalent if $\sigma_{1}=\sigma_{2}$ and $f_{2} \circ f_{1}^{-1}$ is homotopic to a conformal automorphism of $R_{\sigma_{1}}=R_{\sigma_{2}}$. Here the homotopy is considered to be relative to the boundary at infinity $(=r e l . \partial)$ when the corresponding Fuchsian group is of the second kind. A distance between equivalence classes $p_{1}=\left[f_{1}, \sigma_{1}\right]$ and $p_{2}=\left[f_{2}, \sigma_{2}\right]$ in $T(R)$ is defined by $d\left(p_{1}, p_{2}\right)=\log K(h)$, where $h$ is an extremal quasiconformal homeomorphism in the sense that its maximal dilatation $K(h)$ is

Received by the editors September 17, 2003.

2000 Mathematics Subject Classification. Primary 30F60; Secondary 32G15.

Key words and phrases. Teichmüller space, quasiconformal mapping class group, hyperbolic Riemann surface, pants decomposition.

(c)2004 American Mathematical Society 3119

Reverts to public domain 28 years from publication 
minimal in the homotopy class $(r e l . \partial)$ of $f_{2} \circ f_{1}^{-1}$. Then $d$ is a complete distance on $T(R)$, which is called the Teichmüller distance.

The Teichmüller modular group $\operatorname{Mod}(R)$ (or the quasiconformal mapping class group) of $R$ is a group of the homotopy classes (rel. $\partial$ ) of quasiconformal automorphisms of $R$. An element $g$ of $\operatorname{Mod}(R)$ acts on $T(R)$ in such a way that $[f, \sigma] \mapsto\left[f \circ g^{-1}, \sigma\right]$, where $g$ also denotes a representative of the homotopy class. It is evident from definition that $\operatorname{Mod}(R)$ acts on $T(R)$ isometrically with respect to the Teichmüller distance. One can consult a monograph [6] for basic facts on Teichmüller spaces.

We consider a Riemann surface $R$ such that the Teichmüller modular group $\operatorname{Mod}(R)$ consists only of countably many elements. As necessary conditions for $R$ to have this property, we easily see the following.

Proposition 1. If $\operatorname{Mod}(R)$ is countable, then the Riemann surface $R=\mathbb{H}^{2} / \Gamma$ satisfies the following conditions:

(1) The number of simple closed geodesics on $R$ whose lengths are uniformly bounded is finite.

(2) $R$ has no ideal boundary at infinity, that is, the corresponding Fuchsian group $\Gamma$ is of the first kind.

Proof. (1) First we note that the number of closed geodesics having uniformly bounded lengths and having non-empty intersection with a compact subset $B$ of $R$ is finite. Indeed, if infinite, then by assigning a unit directional vector based on a point in $B$ to each closed geodesic, we have infinitely many elements of a compact subset in the unit tangent bundle over $R$. This implies that there is a convergent sequence of axes in $\mathbb{H}^{2}$ for the corresponding elements $\gamma$ of the Fuchsian group $\Gamma$ representing $R$. Moreover, uniform boundedness of the lengths of the closed geodesics enables us to choose a subsequence satisfying the translation lengths of $\gamma$ are also convergent. However, this contradicts the discreteness of $\Gamma$.

Assume that there exist infinitely many simple closed geodesics whose lengths are uniformly bounded. We can choose mutually disjoint $\left\{c_{n}\right\}_{n=1}^{\infty}$ from them, for otherwise, infinitely many such geodesics must intersect a single one, contradicting the claim in the above paragraph. Then the Dehn twist $\chi_{n}$ along each $c_{n}$ is an element of $\operatorname{Mod}(R)$ with uniformly bounded maximal dilatation. Also any infinite composition chosen from $\left\{\chi_{n}\right\}_{n=1}^{\infty}$ gives an element of $\operatorname{Mod}(R)$. See [7]. Since such choices are uncountably many, so are the elements of $\operatorname{Mod}(R)$.

(2) Assume that there exists ideal boundary $\partial R$ at infinity. Then there are uncountable many (actually a continuous family of) quasiconformal automorphisms of $R$ that have different boundary maps on $\partial R$ but freely homotopic to the identity. They define uncountably many distinct elements of $\operatorname{Mod}(R)$.

In Section 3, we construct a Riemann surface $R$ that satisfies these conditions (1) and (2). A proof that $R$ satisfies (2) is a crucial point of our argument, which is given in Section 4 . Then $R$ becomes naturally the required surface. A proof that $\operatorname{Mod}(R)$ is countable is given in Section 5 .

Condition (1) and the following proposition due to Wolpert 9 impose strong restriction on the elements of $\operatorname{Mod}(R)$.

Proposition 2. Let $c$ be a simple closed geodesic on a Riemann surface $R$ with the length $\ell(c)$ and $f: R \rightarrow R^{\prime}$ a quasiconformal homeomorphism onto another 
Riemann surface $R^{\prime}$ with the maximal dilatation $K \geq 1$. Then the geodesic length $\ell^{\prime}(f(c))$ of the free homotopy class of $f(c)$ on $R^{\prime}$ satisfies

$$
\frac{1}{K} \ell(c) \leq \ell^{\prime}(f(c)) \leq K \ell(c) \text {. }
$$

\section{§2. Discontinuity}

In this section, which is not directly related to the construction of our example though, we show that any Teichmüller space $T(R)$ with countable $\operatorname{Mod}(R)$ has a similar property to finite-dimensional Teichmüller spaces. The main result of this section is Theorem 1 , where we show that if $\operatorname{Mod}(R)$ is countable it necessarily acts discontinuously on $T(R)$. We say that the action of $\operatorname{Mod}(R)$ is discontinuous if, for every point $p \in T(R)$, there exists a neighborhood $U$ of $p$ such that the number of elements $g \in \operatorname{Mod}(R)$ satisfying $g(U) \cap U \neq \emptyset$ is finite. Since Teichmüller modular groups of analytically finite Riemann surfaces are necessarily countable, Theorem 1 generalizes the well-known fact that such groups act discontinuously (cf. [6]).

Lemma 1. Let $p=[f, \sigma]$ be a point of the Teichmüller space $T(R)$ and let $R_{\sigma}$ be the corresponding Riemann surface. If the number of simple closed geodesics on $R_{\sigma}$ whose lengths are less than some positive constant $L$ is positive finite, then the orbit $G(p)$ under the Teichmüller modular group $G=\operatorname{Mod}(R)$ is a closed set. Moreover, the isotropy subgroup $\operatorname{Stab}_{G}(p)$, which is isomorphic to the group $\operatorname{Aut}\left(R_{\sigma}\right)$ of all biholomorphic automorphisms of $R_{\sigma}$, is finite.

Proof. Suppose that a sequence of points $p_{n}=\left[f_{n}, \sigma\right]$ in the orbit $G(p)$ converges to a point $q=\left[f_{\infty}, \tau\right] \in T(R)$. Then we may choose $f_{n}$ and $f_{\infty}$ so that the maximal dilatations $K\left(f_{n} \circ f_{\infty}^{-1}\right)$ of $f_{n} \circ f_{\infty}^{-1}: R_{\tau} \rightarrow R_{\sigma}$ converge to 1 . It follows from Proposition 2 that there exists a simple closed geodesic $c$ on $R_{\tau}$ whose length is less than $L$. Also, for any sufficiently large $n, f_{n} \circ f_{\infty}^{-1}$ sends $c$ to one of the finitely many simple closed geodesics on $R_{\sigma}$ with length less than $L$. Hence a subsequence of $f_{n} \circ f_{\infty}^{-1}$ converges locally uniformly to a quasiconformal homeomorphism $h$ : $R_{\tau} \rightarrow R_{\sigma}$. Eventually $K(h)=1$, that is, $h$ is conformal and $\sigma=\tau$. This implies that $q$ belongs to the orbit $G(p)$.

For the latter assertion, we have only to assume all $p_{n}$ and $q$ are coincident with $p$ in the above proof. Then we may regard $f_{n} \circ f_{\infty}^{-1}$ as conformal automorphisms of $R_{\sigma}$. They have a convergent subsequence as we have seen above, while $\operatorname{Aut}\left(R_{\sigma}\right)$ is discrete. This means that $\operatorname{Stab}_{G}(p)$ consists only of finitely many elements.

Remark. For the first statement in Lemma 1, the finiteness of simple closed geodesics with bounded length is necessary. In fact, there is an example of a Teichmüller space $T(R)$ in which the orbit $G(p)$ under $G=\operatorname{Mod}(R)$ is not closed for some $p \in T(R)$.

Theorem 1. If $\operatorname{Mod}(R)$ is countable, then it acts on $T(R)$ discontinuously.

Proof. Suppose that the action of $G=\operatorname{Mod}(R)$ is not discontinuous. Then either (a) there exists a point $p \in T(R)$ such that the isotropy $\operatorname{subgroup~} \operatorname{Stab}_{G}(p)$ is infinite or (b) there exists a point $p \in T(R)$ and a sequence of elements $\left\{g_{n}\right\}$ of $G$ such that $p_{n}=g_{n}(p)$ are different from $p$ and converge to $p$ as $n \rightarrow \infty$. See Fujikawa [5].

By Propositions 1 and 2, for $p=[f, \sigma]$, there exists a positive constant $L$ such that the number of simple closed geodesics on $R_{\sigma}$ whose lengths are less than $L$ is positive finite. Then condition (a) is impossible by Lemma 1. If condition (b) 

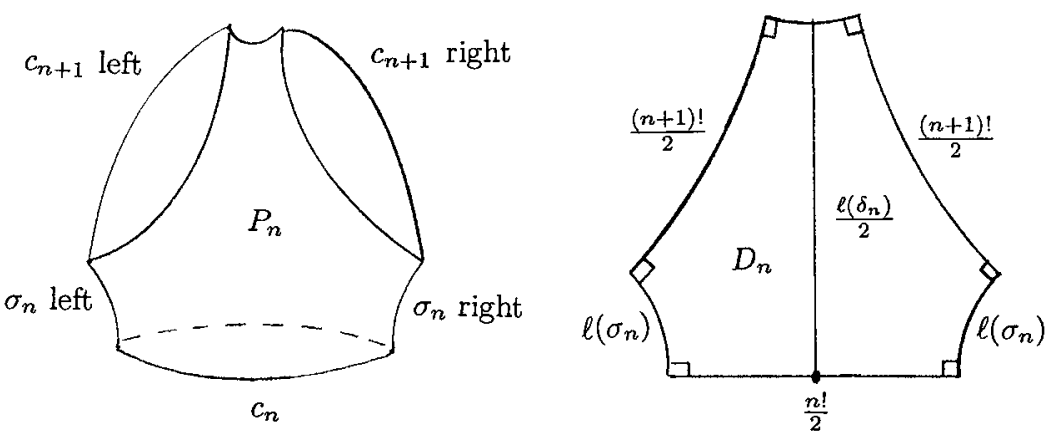

FiguRE 1. A pair of pants and the right-angled hexagon

holds, then $p$ is a point of accumulation of the orbit $G(p)$. By the group invariance, this implies that every point of $G(p)$ is a point of accumulation. Since $G(p)$ is closed by Lemma $1, G(p)$ is a closed perfect set. Since a closed perfect set in a complete metric space is uncountable, $G(p)$ is uncountable and so is $G$. This contradicts the assumption.

\section{$\S 3$. Construction}

The fundamental piece of our construction is a pair of pants $P$, which is a hyperbolic surface with geodesic boundaries $c$ and is homeomorphic to a threepunctured sphere. Every pair of pants admits the canonical orientation-reversing isometric involution. The fixed point set of this involution consists of three geodesic segments $\sigma$, which we call the symmetry axes. Cutting along the symmetry axes, we have two congruent right-angled hexagons $D$.

Let $P_{0}$ be a pair of pants the lengths of whose geodesic boundary components are 0 ! and 1 ! and 1 !. Let $P_{1}$ be a pair of pants with the lengths 1 ! and 2 ! and 2 !. In the same way, for every non-negative integer $n$, let $P_{n}$ be a pair of pants with the lengths $n$ ! and $(n+1)$ ! and $(n+1)$ !. The three symmetry axes divide $P_{n}$ into two congruent right-angled hexagons $D_{n}$. The geodesic boundary components of length $n$ ! and $(n+1)$ ! in $P_{n}$ are denoted by $c_{n}$ and $c_{n+1}$, respectively. The two symmetry axes of $P_{n}$ connecting $c_{n}$ and $c_{n+1}$ are denoted by $\sigma_{n}$. See Figure 1 .

We prepare $2^{n+1}$ copies of $P_{n}$ and glue the geodesic boundary components as follows: Take 2 copies of $P_{0}$ and glue the geodesic boundary component $c_{0}$ of each $P_{0}$ together so that the symmetry axes $\sigma_{0}$ of both $P_{0}$ meet. The resulting hyperbolic surface with 4 geodesic boundary components $c_{1}$ is denoted by $R_{1}$. Next take 4 copies of $P_{1}$ and glue the geodesic boundary component $c_{1}$ of each $P_{1}$ with the 4 boundary components of $R_{1}$ so that the symmetry axes $\sigma_{1}$ of $P_{1}$ meet the symmetry axes of $P_{0}$. The resulting hyperbolic surface with 8 geodesic boundary components $c_{2}$ is denoted by $R_{2}$.

Continuing this process, we obtain, for every positive integer $n$, a hyperbolic surface $R_{n}$ with $2^{n+1}$ geodesic boundary components $c_{n}$ made of $R_{n-1}$ and $2^{n}$ copies of $P_{n-1}$. Then take the exhaustion of these surfaces $R_{n}$, which is $R^{\prime}=\bigcup_{n=1}^{\infty} R_{n}$. In other words, $R^{\prime}$ admits a pants decomposition whose dual graph is the trivalent regular tree. However, this is not yet our required Riemann surface. The reason why not is that $R^{\prime}$ is not complete, which is equivalent to saying that a complete 
hyperbolic surface containing $R^{\prime}$ as a deformation retract has ideal boundary at infinity.

At each step, the symmetry axes of $P_{n}$ and $P_{n+1}$ meet and hence they all together constitute geodesic lines $\{\sigma\}$ in $R^{\prime}$. We measure the length of each $\sigma$. In each pair of pants $P_{n}(n \geq 0)$, the lengths of the three symmetry axes are calculated by trigonometry on the right-angled hexagon $D_{n}$. See Buser [2, Chap. 2] and Figure 1.

Proposition 3. Let $\ell\left(\sigma_{n}\right)$ be the length of the symmetry axes $\sigma_{n}$ of $P_{n}$ connecting the boundary components $c_{n}$ and $c_{n+1}$. Then

$$
\operatorname{arcsinh}\left\{\frac{1}{\sinh (n ! / 4)}\right\}<\ell\left(\sigma_{n}\right)<2 \operatorname{arcsinh}\left\{\frac{1}{2 \sinh (n ! / 4)}\right\} .
$$

Proof. By trigonometry on a right-angled hexagon, we have

$$
\ell\left(\sigma_{n}\right)=\operatorname{arccosh}\left\{\frac{\cosh ((n+1) ! / 2)+\cosh (n ! / 2) \cosh ((n+1) ! / 2)}{\sinh (n ! / 2) \sinh ((n+1) ! / 2)}\right\} .
$$

Since

$$
g(\xi)=\frac{\cosh (\xi)+\cosh (n ! / 2) \cosh (\xi)}{\sinh (n ! / 2) \sinh (\xi)}
$$

is a monotone decreasing function of $\xi$, we have

$$
\begin{aligned}
\operatorname{arcsinh}\left\{\frac{1}{\sinh (n ! / 4)}\right\} & \operatorname{arccosh}\left\{\frac{1+\cosh (n ! / 2)}{\sinh (n ! / 2)}\right\}<\ell\left(\sigma_{n}\right)<\operatorname{arccosh}\left\{\frac{\cosh (n ! / 2)+\cosh ^{2}(n ! / 2)}{\sinh ^{2}(n ! / 2)}\right\} \\
= & 2 \operatorname{arcsinh}\left\{\frac{\cosh (n ! / 4)}{\sinh (n ! / 2)}\right\} \\
= & 2 \operatorname{arcsinh}\left\{\frac{1}{2 \sinh (n ! / 4)}\right\} .
\end{aligned}
$$

This is the desired estimate.

The right-hand side of this inequality is further estimated from above by

$$
2 \operatorname{arcsinh} \frac{1}{2 \sinh (n ! / 4)}<\frac{1}{\sinh (n ! / 4)} .
$$

Hence the sum of $\ell\left(\sigma_{n}\right)$ taken over all $n$ converges, which implies that the length of every $\sigma$ is finite. Therefore $R^{\prime}=\bigcup_{n=1}^{\infty} R_{n}$ is not complete.

In order to obtain a complete hyperbolic surface we twist $R^{\prime}$ along each simple closed geodesic $c_{n}$. By twisting the appropriate amount we can make the distance between $c_{0}$ and $P_{n}$ large. See Basmajian [1. Note that since the trivalent regular tree corresponding to $R^{\prime}$ has uncountably many topological ends, so does $R^{\prime}$. In order that the surface obtained by twisting $R^{\prime}$ be complete, the twisting along each component of every $c_{n}$ should be in some sense given equal weight so that ideal boundary components at infinity, which are at most countable, do not appear. However, this intuition does not tell explicitly how much to twist, and it is a subtle problem to determine the right amount. For example if we apply a $1 / 4$-twist along each $c_{n}$, we do not know whether the resulting surface is complete.

We now proceed to the construction, and define the twists as follows. See Figure 2 . For every $P_{n}$, we take a point $x_{n}$ on the boundary component $c_{n}$ so that it is the 


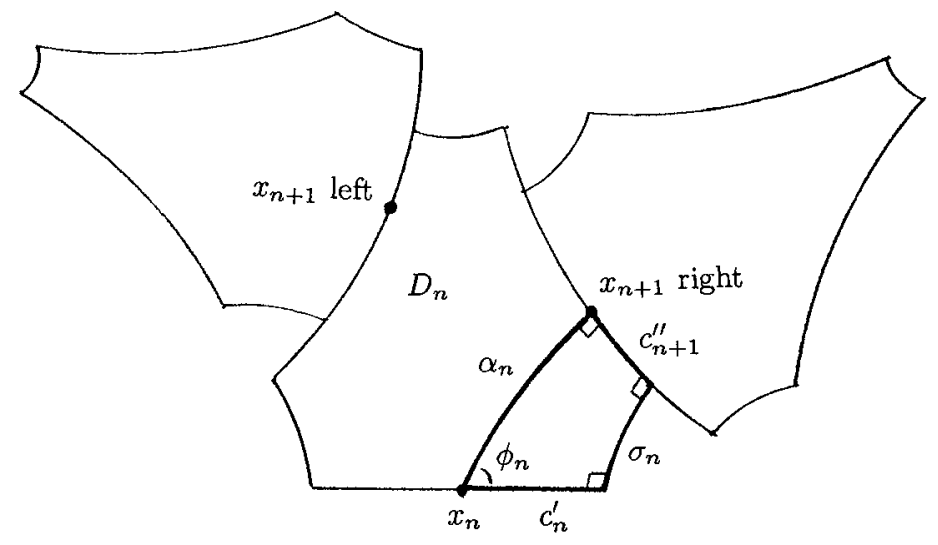

FIgURE 2. Twist and the trirectangle

midpoint between the symmetry axes and call it the center of $P_{n}$. Starting from $x_{n}$, we draw the shortest geodesic segment $\alpha_{n}$ in $P_{n}$ to the boundary component $c_{n+1}$ right; $\alpha_{n}$ and $c_{n+1}$ right intersect in the right angle. Along the $c_{n+1}$ right, we twist $P_{n+1}$ so that the center $x_{n+1}$ of $P_{n+1}$ comes to the endpoint of $\alpha_{n}$. Next, along the $c_{n}$ left, we also twist $P_{n+1}$ left in the same direction and by the same amount as $P_{n+1}$ right. Then the center $x_{n+1}$ of $P_{n+1}$ left comes to a certain point on the boundary component $c_{n+1}$ left. The geodesic segment in $P_{n}$ connecting $x_{n}$ and this new place for $x_{n+1}$ left is denoted by $\beta_{n}$. Along $c_{0}$ between the two $P_{0}$, we give no twist. Namely, the two centers of the two $P_{0}$ sit on the same point.

From the following proposition, we can see that the length of the geodesic segment $\alpha_{n}$ in $P_{n}$ is uniformly bounded away from zero for every $n \geq 0$. Also the intersection of $\alpha_{n}$ and $c_{n+1}$ (the new place for $x_{n+1}$ right) is always on the right side of the midpoint of $c_{n+1}$. The latter implies that all the directions of the twists are the same; $P_{n+1}$ turns to the right if we observe it from $P_{n}$.

Proposition 4. In each pair of pants $P_{n}(n \geq 0)$, consider a trirectangle made of the geodesic segments $\alpha_{n}, \sigma_{n}, c_{n}^{\prime}$ and $c_{n+1}^{\prime \prime}$, where $c_{n}^{\prime}$ and $c_{n+1}^{\prime \prime}$ are the portions of $c_{n}$ and $c_{n+1}$ (Figure 2$)$. Let $\ell(\cdot)$ denote the length of each segment, and $\phi_{n}$ the angle at $x_{n}$ between $c_{n}^{\prime}$ and $\alpha_{n}$. Then $\ell\left(\alpha_{n}\right)>\operatorname{arcsinh} 1$ and $\ell\left(c_{n+1}^{\prime \prime}\right)<n ! / 4$ for every $n \geq 0$. Moreover, $\phi_{n}>\pi / 4$.

Proof. A trirectangle is determined by the lengths of two sides and we know that $\ell\left(c_{n}^{\prime}\right)=n ! / 4$ and $\sinh \ell\left(\sigma_{n}\right)>1 / \sinh (n ! / 4)$ by Proposition 3. The other lengths are obtained from the following formulas $([2])$ :

$$
\begin{aligned}
\sinh \ell\left(\alpha_{n}\right) & =\sinh \ell\left(\sigma_{n}\right) \cosh \ell\left(c_{n}^{\prime}\right), \\
\tanh \ell\left(c_{n+1}^{\prime \prime}\right) & =\frac{1}{\cosh \ell\left(\sigma_{n}\right)} \tanh \ell\left(c_{n}^{\prime}\right)<\tanh \ell\left(c_{n}^{\prime}\right) .
\end{aligned}
$$

From the first formula, we have

$$
\sinh \ell\left(\alpha_{n}\right)>\frac{1}{\sinh (n ! / 4)} \cosh (n ! / 4)>1 .
$$

From the second formula, we have $\ell\left(c_{n+1}^{\prime \prime}\right)<n ! / 4$. 
The angle $\phi_{n}$ is also obtained from the following ([2]):

$$
\tan \phi_{n}=\frac{\cosh \ell\left(\sigma_{n}\right)}{\sinh (n ! / 4) \sinh \ell\left(\sigma_{n}\right)} .
$$

Then, by simple calculation as in Proposition 3, this is estimated from below by

$$
\frac{1+\cosh (n ! / 2)}{\sinh (n ! / 2) \sqrt{1+(2 \sinh (n ! / 4))^{-2}}}>1 .
$$

Hence we have $\phi_{n}>\pi / 4$.

Remark. (1) By estimating from the opposite side and taking the limit, we see that the constants obtained in the above proposition are sharp: $\lim _{n \rightarrow \infty} \ell\left(\alpha_{n}\right)=$ $\operatorname{arcsinh} 1$ and $\lim _{n \rightarrow \infty} \phi_{n}=\pi / 4$. (2) Since $\ell\left(c_{n+1}^{\prime \prime}\right)<n ! / 4$, the rotation number of the twist is at least $n / 4(n+1)$, which converges to $1 / 4$ as $n \rightarrow \infty$.

We denote the exhaustion $\bigcup_{n=1}^{\infty} R_{n}$ with the twists along $c_{n}$ by $R$. In the next section, we prove that $R$ is a complete hyperbolic surface.

\section{$\S 4$. Completeness}

In order to prove that $R$ is complete, we suppose to the contrary that $R$ is not complete. Then, since $R=\bigcup_{n=1}^{\infty} R_{n}$ has the exhaustion by the hyperbolic surfaces $R_{n}$ with geodesic boundaries, any boundary component of $R$ is geodesic. We develop $R$ to the hyperbolic plane $\mathbb{H}^{2}$ to obtain a simply connected domain $\tilde{R}$. Then $\tilde{R}$ is bounded by countably many geodesic lines $L$ in $\mathbb{H}^{2}$ together with ideal boundary at infinity. For each geodesic line $L$, a sequence $\left\{\tilde{c}_{n}\right\}$ of geodesic lines in $\mathbb{H}^{2}$ converges to $L$, where $\tilde{c}_{n}$ is a developed image of a simple closed geodesic $c_{n}$ in $R$.

We choose a fundamental domain of $R$ as follows. In each pair of pants $P_{n}$, take the right-angled hexagon $D_{n}$ containing the center $x_{n}$ among the congruent two. Since the center $x_{n+1}$ of $P_{n+1}$ is on the boundary of $D_{n}$, the union of all the $D_{n}$ constitutes a simply connected domain $D$ such that $D$ and the other congruent half $D^{\prime}$ together make $R$. We develop $D$ and $D^{\prime}$ into $\mathbb{H}^{2}$ as simply connected domains, which are denoted by $\tilde{D}$ and $\tilde{D}^{\prime}$. The union of $\tilde{D}$ and $\tilde{D}^{\prime}$ makes a fundamental domain of $R$. Since they are congruent, it is enough to consider only $\tilde{D}$.

We estimate the least distance necessary to cross over $\tilde{D}$, which is the infimum of the distances between any two distinct components of the complement of $\tilde{D}$ in $\tilde{R}$. It is easy to see that we have only to measure a path along $\tilde{c}_{n}$, whose length is bounded from below by $n ! / 4$. Hence the distance for crossing over $\tilde{D}$ is at least $1 / 4$.

Take a geodesic line $L$ that is a boundary component of $\tilde{R}$. We draw an arc $\tilde{\eta}$ of finite length from a point inside $\tilde{R}$ to an interior point of $L$. (The role of $\tilde{\eta}$ is not important; it is merely for convenience of explanation.) There are two cases to be considered: $\tilde{\eta}$ crosses over either infinitely many orbits of $\tilde{D}$ or $\tilde{D}^{\prime}$ under the holonomy group, or only finitely many of them. In the former case, $\tilde{\eta}$ would contain infinitely many subarcs of length greater than $1 / 4$, which contradicts the fact that the length of $\tilde{\eta}$ is finite. In the latter case, without loss of generality, we may assume that $\tilde{\eta}$ starts from $\tilde{x}_{0}$ and lies entirely in $\tilde{D}$, where $\tilde{x}_{0} \in \tilde{D}$ corresponds to $x_{0} \in R$. This means that the closure of $\tilde{D}$ has the intersection with $L$ in $\mathbb{H}^{2}$ and 


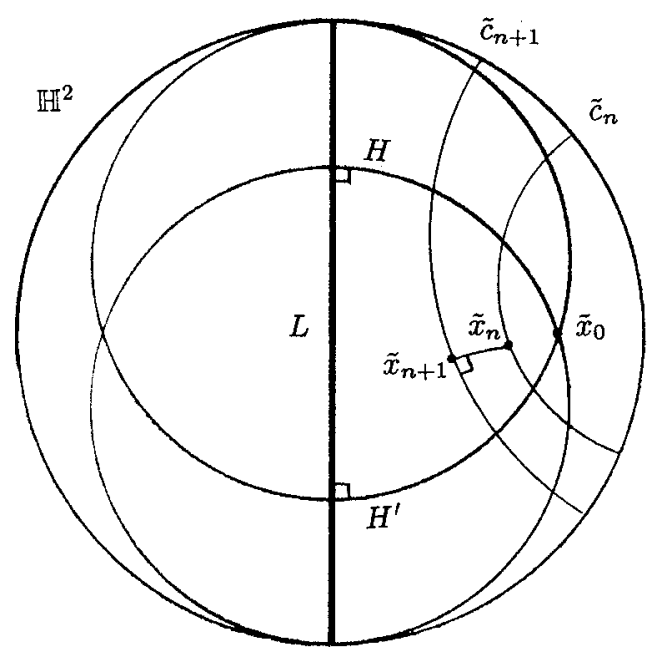

Figure 3. Bounded by horocircles

$\tilde{\eta}$ lands on $L$ after passing through the geodesic lines $\tilde{c}_{n}$ for all $n$. At each level $n$, $\tilde{\eta}$ goes choosing one of the left and the right $\tilde{c}_{n+1}$ towards $L$.

We consider an infinite tree $T$ based on $x_{0}$ that consists of the geodesic segments $\alpha_{n}$ and $\beta_{n}(n \geq 0)$. Any path in $T$ towards infinity is determined by choosing one of $\alpha_{n}$ and $\beta_{n}$ (equivalently choosing one of the next vertices $x_{n+1}$ ) at each vertex $x_{n}$. We take a path $\gamma$ in $T$ that has the same itinerary as the arc $\tilde{\eta}$. Let $\tilde{\gamma} \subset \tilde{D}$ be the developed image of $\gamma$. This is a piecewise geodesic ray starting from $\tilde{x}_{0}$ and connecting $\tilde{x}_{1}, \tilde{x}_{2}, \ldots$ consecutively. For each $n, \tilde{\eta}$ and $\tilde{\gamma}$ intersect the same geodesic line $\tilde{c}_{n}$ where the $\tilde{x}_{n}$ lies.

We consider possibility of the existence of the path $\gamma$ constructed above. The essential case in our arguments is when $\gamma$ is the $\alpha$-path, which is the piecewise geodesic ray $\alpha_{0} \cup \alpha_{1} \cup \alpha_{2} \cdots$. We can see that the length of the $\alpha$-path is infinite by Proposition 4. However, the following Lemma 2 with Figure 3 shows that if $\gamma$ is the $\alpha$-path, then its length must be finite. Thus the possibility that $\gamma$ is the $\alpha$-path is eliminated.

Lemma 2. Let $L$ be a geodesic line and $\tilde{x}_{0} \notin L$ a point in the hyperbolic plane $\mathbb{H}^{2}$. Suppose that a sequence of mutually disjoint geodesic lines $\left\{\tilde{c}_{n}\right\}_{n=1}^{\infty}$ separates $L$ from $\tilde{x}_{0}$ consecutively: $\tilde{c}_{1}$ separates $L$ from $\tilde{x}_{0}$, and inductively $\tilde{c}_{n+1}$ separates $L$ from $\tilde{c}_{n}$. Let $\tilde{x}_{1} \in \tilde{c}_{1}$ be the nearest point from $\tilde{x}_{0}$, and inductively let $\tilde{x}_{n+1} \in \tilde{c}_{n+1}$ be the nearest point from $\tilde{x}_{n}$. Then the sum of the distances $\sum_{n=0}^{\infty} d\left(\tilde{x}_{n}, \tilde{x}_{n+1}\right)$ is bounded from above by twice the length from $\tilde{x}_{0}$ to $L$ along a horocircle tangent at the endpoint of $L$. In particular, the sum converges.

Proof. Let $H$ and $H^{\prime}$ be the horocircles tangent at the endpoints of $L$ and passing through $\tilde{x}_{0}$. For a point $z \in \tilde{c}_{n}$ within the region bounded by $H$ and $H^{\prime}$, consider the distance $f(z)=d\left(z, \tilde{c}_{n+1}\right)$ between $z$ and $\tilde{c}_{n+1}$ as a continuous function of $z$. It takes its maximum at one of the endpoints of the segment in $\tilde{c}_{n}$ bounded by $H$ and $H^{\prime}$. Since the maximum is further bounded by the arclength along $H$ or $H^{\prime}$ 


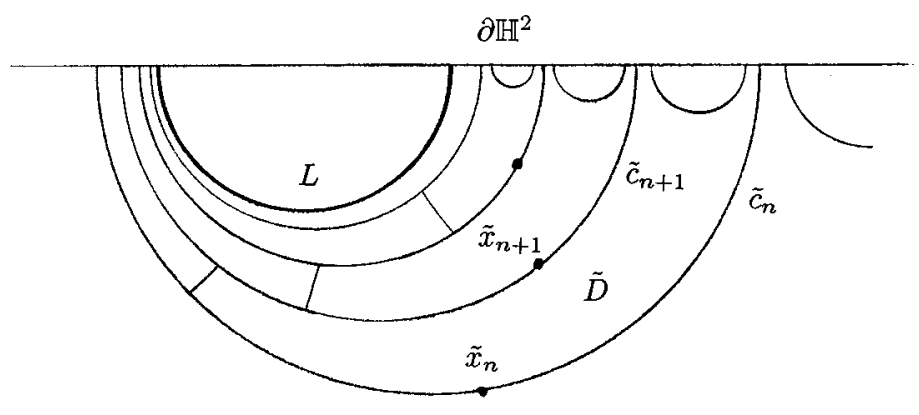

Figure 4 . The $\beta$-path goes right

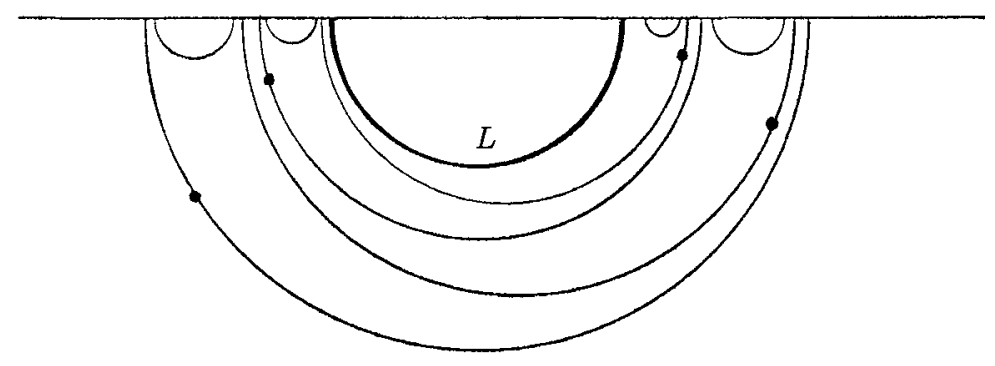

Figure 5. Alternate $\alpha$ - and $\beta$-segments

between $\tilde{c}_{n}$ and $\tilde{c}_{n+1}$, so is $d\left(\tilde{x}_{n}, \tilde{x}_{n+1}\right)$. Taking the sum over all $n$, we have the assertion.

Next we consider the case when $\gamma$ is the $\beta$-path, which is the piecewise geodesic ray $\beta_{0} \cup \beta_{1} \cup \beta_{2} \cdots$. We can also eliminate this possibility as follows. Along each $\tilde{c}_{n}$ $(n \geq 1)$, the adjacent hexagon shifts to the right at least $n ! / 4-(n-1) ! / 4$ length by Proposition 4 . Since $\tilde{c}_{n}$ sit nearly parallel to $L$ for sufficiently large $n$, this forces the $\operatorname{arc} \tilde{\eta}$ in $\tilde{D}$ to land at $\partial \mathbb{H}^{2}$ on the right side of $L$, not at an interior point of $L$. See Figure 4. (To give another reason why this case cannot occur, we have only to notice that the other $\alpha$-path in the backside $D^{\prime}$ exists in parallel to the $\beta$-path in $D$ and they together travel passing through the same $c_{n}$. Since the $\alpha$-path cannot behave like this as is seen above, $\gamma$ cannot be the $\beta$-path.)

The case when $\gamma$ contains finitely many $\alpha$ - or $\beta$-segments is excluded in the same reason as above. The remaining possibility is that $\gamma$ contains both $\alpha$ - and $\beta$-segments infinitely many. By Proposition 4 , each $\alpha_{n}$ makes at least angle $\pi / 4$ measured from $c_{n}$. Since $\beta$-segments are given by turning the $x_{n+1}$ left to the right, the angle made by $\beta_{n}$ and $c_{n}$ is greater than the one made by $\alpha_{n}$, namely, it is also greater than $\pi / 4$. An elementary geometric observation shows that, for all sufficiently large $n$, the base point $\tilde{x}_{n}$ of an $\alpha_{n}$ in $\tilde{\gamma}$ must be close to the left endpoint of $L$, and the base point of a $\beta_{n}$ in $\tilde{\gamma}$ must be close to the right endpoint of $L$. Then both the endpoints of $L$ are accumulation points of $\tilde{\gamma}$. However, this is impossible for the reason that outgoing angles of $\tilde{\gamma}$ at the intersection with $\tilde{c}_{n}$ are uniformly bounded below by $\pi / 4$. (We may alternatively use the fact $\lim _{n \rightarrow \infty} \ell\left(\alpha_{n}\right)=\operatorname{arcsinh} 1$ for this reasoning.) See Figure 5 . 
From the arguments in this section, we conclude:

Theorem 2. The hyperbolic surface $R$ defined in Section 3 is complete.

\section{$\S 5$. Countability}

In this section, we prove that the Teichmüller modular $\operatorname{group} \operatorname{Mod}(R)$ for our Riemann surface $R$ consists of countably many elements.

Proposition 5. In the pair of pants $P_{n}$ of $R$, consider the shortest geodesic arc $\delta_{n}\left(\not \subset c_{n}\right)$ that connects the boundary component $c_{n}$ to itself. Then its hyperbolic length $\ell\left(\delta_{n}\right)$ satisfies

$$
\ell\left(\delta_{n}\right)=2 \operatorname{arcsinh}\left\{\frac{\cosh ((n+1) ! / 2)}{\sinh (n ! / 4)}\right\}>n ! \times n .
$$

Proof. By symmetry, it is easy to see that $\delta_{n}$ is an arc starting from the center $x_{0}$ perpendicularly to the backside center on $c_{n}$. Using trigonometry on the rightangled hexagon $D_{n}$ or trigonometry on the right-angled pentagon, we can calculate the half length of $\delta_{n}$. See Figure 1.

Based on this proposition, we have the following.

Lemma 3. (1) The hyperbolic length of a closed geodesic contained in $R-\overline{R_{n}}$ is greater than $(n+1) !>n ! \times n .(2)$ The hyperbolic length of a closed geodesic $\left(\neq c_{n}\right)$ having intersection with $\partial R_{n}$ is greater than $n ! \times n$.

Proof. Every closed geodesic contained in $R-\overline{R_{n}}$ is either coincident with $c_{n+i}$ for some $i \geq 1$ or containing a subarc in $P_{n+i}$ for some $i \geq 1$ with both endpoints on $c_{n+i}$. In the former case, the length is $(n+i)$ !, and in the latter case the length is greater than $(n+i) ! \times(n+i)$ by Proposition 5 . In both cases, it is greater than $(n+1)$ !. Similarly, every closed geodesic $\left(\neq c_{n}\right)$ having intersection with $\partial R_{n}$ contains a subarc in $P_{n+i}$ for some $i \geq 0$ with both endpoints on $c_{n+i}$. Hence its length is greater than $n ! \times n$.

We consider quasiconformal automorphisms of $R$. Proposition 2 gives strong restriction to the possibility of the images of $R_{n}$.

Lemma 4. Let $g: R \rightarrow R$ be a K-quasiconformal automorphism of $R$. Then, for every $n \geq K$, the image $g\left(R_{n}\right)$ of the subdomain $R_{n}$ is freely homotopic to $R_{n}$ in $R$.

Proof. If not, there exists a geodesic boundary component $c_{n}$ of $\partial R_{n}$ such that $g\left(c_{n}\right)$ is freely homotopic to a simple closed geodesic either contained in $R-\overline{R_{n}}$ or having the intersection with $\partial R_{n}$. By Lemma 3 , the geodesic length $\ell\left(g\left(c_{n}\right)\right)$ of the free homotopy class $g\left(c_{n}\right)$ is greater than $n ! \times n$. On the other hand, since $\ell\left(c_{n}\right)=n$ ! and $g$ is $K$-quasiconformal, Proposition 2 asserts that $\ell\left(g\left(c_{n}\right)\right) \leq K \cdot n ! \leq n ! \times n$. This contradiction proves the lemma.

If we apply this lemma to $R_{n}$ for each integer $n \geq K$, we see that any $K$ quasiconformal automorphism of $R$ maps every pair of pants $P_{n}$ homotopically onto a pair of pants of the same size. Our final work is then to eliminate the possibility of Dehn twists along each $c_{n}$ for $n \geq K$. We succeed in doing that and conclude the following. 
Theorem 3. Let $g: R \rightarrow R$ be a $K$-quasiconformal automorphism of $R$. Then, on each connected component $E_{n}$ of $R-\overline{R_{n}}$ for $n \geq \max \{K, 5\}$, the $g$ restricted to $E_{n}$ is homotopic to a conformal homeomorphism of $E_{n}$ onto another connected component of $R-\overline{R_{n}}$.

Proof. We apply Lemma 4 to $R_{n+i}$ for every $i \geq 0$. Then we can see that the image of each pair of pants $P_{n+i}$ under $g$ is homotopic to some other pair of pants $P_{n+i}$ with the same size. This implies that $g$ restricted to each $P_{n+i}$ is homotopic to a conformal homeomorphism. Hence, on each connected component $E_{n}$ of $R-$ $\overline{R_{n}}$ for $n \geq K, g$ is homotopic to a conformal homeomorphism possibly with the composition of half Dehn twists along simple closed geodesics $c_{n+i}$ for $i \geq 0$.

We will prove that $g$ does not cause a half Dehn twist $\chi$ along a simple closed geodesic $c_{n+i}$. The self-composition $\chi^{2}$ is the full Dehn twist along $c_{n+i}$ and the maximal dilatation of any quasiconformal automorphism in the homotopy class of $\chi^{2}$ can be estimated from below as

$$
K\left(\chi^{2}\right) \geq \sqrt{\left\{\frac{(n+i) !}{\pi}\right\}^{2}+1} \geq \frac{n !}{\pi} .
$$

See [7. Since $K(\chi)^{2} \geq K\left(\chi^{2}\right)$, we have $K(\chi) \geq\{n ! / \pi\}^{1 / 2}>K$. This estimate is also valid for any quasiconformal automorphism that is composed by multiple twists along $c_{n+i}$. However, since $g$ is $K$-quasiconformal, no such twist is possible.

As a consequence, we obtain the required property for the Teichmüller modular group.

Corollary 1. The Teichmüller modular group $\operatorname{Mod}(R)$ for the Riemann surface $R$ consists only of countably many elements.

Proof. We have only to see that, for every integer $n \geq 5$, a subset $\operatorname{Mod}(R)_{n}$ consisting of the elements in $\operatorname{Mod}(R)$ that have an $n$-quasiconformal automorphism $g$ as a representative has at most a countable number of elements. By Theorem $3, \operatorname{Mod}(R)_{n}$ is embedded in the reduced Teichmüller modular group $\operatorname{Mod}^{\#}\left(R_{n}\right)$, which is the group of the free homotopy classes of (quasiconformal) automorphisms of the bordered surface $R_{n}$. Since $R_{n}$ is topologically finite, $\operatorname{Mod}^{\#}\left(R_{n}\right)$ is finitely generated and in particular countable. Hence so is $\operatorname{Mod}(R)_{n}$. (In fact, it consists of finitely many elements.)

\section{§6. BY-PRODUCTS}

The Riemann surface $R$ constructed in the previous sections has distinguished properties other than the countability of $\operatorname{Mod}(R)$. We note the following consequences as corollaries to Theorem 3 and Lemma 3.

An asymptotically conformal homeomorphism $f: R \rightarrow R^{\prime}$ is a quasiconformal homeomorphism having a property that, for every $\epsilon>0$, there exists a compact subset $V$ of $R$ such that the maximal dilatation of $f$ restricted to $R-V$ is less than $1+\epsilon$. This concept plays a central role in the theory of asymptotic Teichmüller spaces developed by Earle, Gardiner and Lakic [4], 6, Chap. 14]. The asymptotically conformal mapping class group $\operatorname{Mod}_{0}(R)$ is defined to be a subgroup of $\operatorname{Mod}(R)$ consisting of all homotopy classes that have an asymptotically conformal automorphism of $R$ as a representative. For an analytically finite Riemann surface $R$, it is clear that $\operatorname{Mod}_{0}(R)$ is coincident with $\operatorname{Mod}(R)$. Theorem 3 implies that 
our Riemann surface $R$ gives an example of infinite topological type that holds this property.

Corollary 2. The asymptotically conformal mapping class group $\operatorname{Mod}_{0}(R)$ is coincident with $\operatorname{Mod}(R)$ for the Riemann surface $R$.

The asymptotic Teichmüller space $A T(R)$ is the Teichmüller space $T(R)$ modulo the subspace $T_{0}(R)$ consisting of the asymptotically conformal Teichmüller classes. For an analytically finite Riemann surface $R$, it is clear that $T(R)=T_{0}(R)$ and hence $A T(R)$ is trivial. When $R$ is not analytically finite, it is proved in [4] that $\operatorname{Mod}(R)$ acts on $A T(R)$ and this action is faithful if and only if $R$ is conformally equivalent to the unit disk or a punctured unit disk. The asymptotically conformal mapping class group $\operatorname{Mod}_{0}(R)$ is the isotropy subgroup of $\operatorname{Mod}(R)$ with respect to the origin of $A T(R)$. Remark that, as the action of $\operatorname{Mod}(R)$ on $A T(R)$ is not transitive, the isotropy subgroups are not necessarily conjugate to each other. Corollary 2 says that our Riemann surface $R$ gives an example where every element of $\operatorname{Mod}(R)$ fixes the origin of $A T(R)$.

Next, for a Riemann surface $R$ with no ideal boundary at infinity, we consider the group of all homotopy classes $g$ of orientation-preserving homeomorphic automorphisms of $R$ and its subgroup $\operatorname{Mod}_{\mathrm{ls}}(R)$ with the following definition: $g$ belongs to $\operatorname{Mod}_{\mathrm{ls}}(R)$ if there exists a constant $\kappa \geq 1$ such that

$$
\frac{1}{\kappa} \ell(c) \leq \ell(g(c)) \leq \kappa \ell(c)
$$

for every simple closed geodesic $c$ on $R$. By Proposition 2, $\operatorname{Mod}(R)$ is a subgroup of $\operatorname{Mod}_{\mathrm{ls}}(R)$. For a topologically finite Riemann surface $R$, they are coincident. If $R$ is not topologically finite, then $\operatorname{Mod}_{\mathrm{ls}}(R)$ always seems uncountable, and this is true for our Riemann surface $R$. Indeed, statement (2) in Lemma 3 says that the length of any simple closed geodesic $c$ intersecting $c_{n}$ is relatively long to the length of $c_{n}$. Hence the Dehn twist along $c_{n}$ does not change the ratio for $c$ so much. Since an arbitrary infinite composition of such Dehn twists belongs to $\operatorname{Mod}_{\mathrm{ls}}(R)$, it contains uncountably many elements. On the other hand, $\operatorname{Mod}(R)$ is countable by Corollary 1. Hence we have the following.

Corollary 3. The Teichmüller modular group $\operatorname{Mod}(R)$ is a proper subgroup of $\operatorname{Mod}_{\mathrm{ss}}(R)$ for the Riemann surface $R$.

On the Teichmüller space $T(R)$, the length spectrum distance between $\left[f_{1}, \sigma_{1}\right]$ and $\left[f_{2}, \sigma_{2}\right]$ is defined by measuring the ratio of the lengths

$$
\sup _{c}\left|\log \frac{\ell_{\sigma_{1}}\left(f_{1}(c)\right)}{\ell_{\sigma_{2}}\left(f_{2}(c)\right)}\right|,
$$

where the supremum is taken over all simple closed geodesics $c$ on $R$. By Proposition 2 again, this is not greater than the usual Teichmüller distance. On the Teichmüller space of an analytically finite Riemann surface, these distances are topologically equivalent. Problems around this fact were studied by T. Sorvali, Z. Li and L. Liu among others.

Recently, Shiga [8] considered a Riemann surface of infinite topological type for which the Teichmüller distance and the length spectrum distance induce different topologies on the Teichmüller space. Our construction of the Riemann surface $R$ is related to his work, however, our requirement for $R$ is much stronger. Actually, 
again from statement (2) in Lemma 3, we see that our Riemann surface $R$ satisfies a condition given in [8] from which the difference of the two distances is deduced.

Corollary 4. On the Teichmüller space $T(R)$ of the Riemann surface $R$, the Teichmüller distance and the length spectrum distance induce different topologies.

\section{REFERENCES}

1. A. Basmajian, Hyperbolic structures for surfaces of infinite type, Trans. Amer. Math. Soc. 336 (1993), 421-444. MR1087051 (93e:30087)

2. P. Buser, Geometry and spectra of compact Riemann surface, Progress in Mathematics 106, Birkhäuser, 1992. MR:1183224 (93g:58149)

3. A. Epstein, Effectiveness of Teichmüller modular groups, In the tradition of Ahlfors and Bers, Contemporary Math. 256, AMS, 2000, pp. 69-74. MR.1759670|(2001a:30059)

4. C. Earle, F. Gardiner and N. Lakic, Teichmüller spaces with asymptotic conformal equivalence, preprint.

5. E. Fujikawa, Limit sets and regions of discontinuity of Teichmüller modular groups, Proc. Amer. Math. Soc. 132 (2004), 117-126. MR2021254 (2004h:30057)

6. F. Gardiner and N. Lakic, Quasiconformal Teichmüller theory, SURV 76, American Mathematical Society, 2000. MR1730906 (2001d:32016)

7. K. Matsuzaki, The infinite direct product of Dehn twists acting on infinite dimensional Teichmüller spaces, Kodai Math. J. 26 (2003), 279-287. MR.2018722(2004k:30110)

8. H. Shiga, On a distance defined by the length spectrum on Teichmüller space, Ann. Acad. Sci. Fenn. 28 (2003), 315-326. MR1996441 (2004i:30043)

9. S. Wolpert, The length spectra as moduli for compact Riemann surfaces, Ann. of Math. 109 (1979), 323-351. MR0528966 (80j:58067)

Department of Mathematics, Ochanomizu University, Otsuka 2-1-1, Bunkyo-ku, TOKYO 112-8610, JAPAN

E-mail address: matsuzak@math.ocha.ac.jp 